\title{
Analytical expressions for commensal-host ecological model: Homotopy perturbation method
}

\author{
Vembu Ananthaswamy ${ }^{1, ~}$, Lucas Sahaya Amalraj ${ }^{2}$ \\ ${ }^{1}$ Department of Mathematics, The Madura College, Madurai, Tamil Nadu, India \\ ${ }^{2}$ Department of Mathematics, Raja Doraisingam Government Arts College, Sivagangai District, Tamil Nadu, India \\ Email address: \\ ananthu9777@rediffmail.com (V. Ananthaswamy)
}

\section{To cite this article:}

Vembu Ananthaswamy, Lucas Sahaya Amalraj. Analytical Expressions for Commensal-Host Ecological Model: Homotopy Perturbation Method. American Journal of Applied Mathematics. Vol. 2, No. 5, 2014, pp. 149-154. doi: 10.11648/j.ajam.20140205.11

\begin{abstract}
In this paper a mathematical commensal-host ecological model with replenishment rate for both species is discussed. This model is characterized by a pair of first order non-linear coupled differential equations. The non-linear coupled system-equations are solved analytically by using Homotopy perturbation method. Further, our results are compared with the previous work and a satisfactory agreement is noted.
\end{abstract}

Keywords: Mathematical Model, Commensal, Host, Replenishment Rate, Non-Linear Differential Equations, Homotopy Perturbation Method

\section{Introduction}

The analytical solutions for the first order non-linear coupled differential equations are not possible directly because of intractability of non-linear terms. Techniques like perturbation to linearizing the non-linear coupled differential equations to give qualitative nature of solutions in treatises of Cushing [3], Phanikumar Kapoor [4], Meyer [6], Phanikumar and Pattabhi Ramacharyulu [7].

The previous investigation presented [1] a four stage recursive procedure to give an approximate solution for a first order non-linear coupled differential equations and same is applied to solve the commensal-host ecological model with replenishment rate for both the species. A similar algorithm was earlier developed by Langlosis and Rivlin [5] to obtain approximate solution for the flows of slightly visco- elastic fluids. Srinivas [8] and Bhaskara Rama Sarma [2] adopted this algorithm for a general two species eco system.

The purpose of this paper is to derive the approximate analytical solution of the populations of the two species for all values of the dimensionless parameters using the Homotopy perturbation method.

\section{The Basic Model Equation}

The model equations are obtained as

$$
\begin{gathered}
\frac{d N_{1}(t)}{d t}=a_{1} N_{1}(t)-a_{11} N_{1}^{2}(t)+a_{12} N_{1}(t) N_{2}(t)+h_{1} \\
\frac{d N_{2}(t)}{d t}=a_{2} N_{2}(t)-a_{22} N_{2}^{2}(t)+h_{1}
\end{gathered}
$$

With the initial conditions

$$
N_{1}(0)=N_{10} ; N_{2}(0)=N_{20}
$$

where $N_{1}(t)$ and $N_{2}(t)$ are the population of the species $S_{1}$ and $S_{2}$ respectively at time $t, a_{1}$ and $a_{2}$ are the natural growth rates of species $S_{1}$ and $S_{2}$ respectively, $a_{11}$ and $a_{22}$ are the rates of decrease of species $S_{1}$ and $S_{2}$ respectively due to limited resources, $a_{12}$ is the inhibition coefficient, $h_{1}$ and $h_{2}$ are the replenishment rates of $S_{1}$ and $S_{2}$ respectively. All the coefficients $a_{1}, a_{2}, a_{11}, a_{12}, a_{22}, h_{1}$ and $h_{2}$ are positive values.

\section{Solution of the Non-Linear Initial Value Problem Using the Homotopy Perturbation Method (HPM)}

Recently, many authors have applied the Homotopy perturbation method (HPM) to solve the non-linear problem in physics and engineering sciences [9-12]. This method is also used to solve some of the non-linear problem in physical 
sciences [13-15]. This method is a combination of Homotopy in topology and classic perturbation techniques. Ji-Huan He used to solve the Lighthill equation [13], the Duffing equation [14] and the Blasius equation [15-16]. The HPM is unique in its applicability, accuracy and efficiency. The HPM uses the imbedding parameter $p$ as a small parameter, and only a few iterations are needed to search for an asymptotic solution. Using this method [17-19], we can obtain solution to the eqns. (1) - (3) as follows:

$$
\begin{aligned}
& N_{1}(t)=\left(k_{0} e^{a_{1} t}-k_{1}\right)+A e^{a_{1} t}+\left[\frac{a_{11} k_{1}^{2}-a_{12} k_{1} u_{1}}{a_{1}}\right]+\left[2 a_{11} k_{0} k_{1}-a_{12} k_{0} u_{1}\right] t e^{a_{1} t} \\
& -\left[\frac{a_{12} k_{1} u_{0}}{a_{2}-a_{1}}\right]-\left[\frac{a_{11} k_{0}^{2} e^{a_{2} t}}{a_{1}}\right]+\left[\frac{a_{12} k_{0} u_{0} e^{\left(a_{1}+a_{2}\right) t}}{a_{2}}\right] \\
& N_{2}(t)=\left(u_{0} e^{a_{2} t}-u_{1}\right)+\left(a_{22} / a_{2}\right)\left[\left(u_{0}^{2}-u_{1}^{2}\right) e^{a_{2} t}-\left(u_{1}^{2}-u_{0}^{2} e^{2 a_{2} t}\right)\right]+2 a_{22} u_{0} u_{1} e^{a_{2} t}
\end{aligned}
$$

where

$$
\begin{gathered}
k_{0}=N_{10}+\frac{h_{1}}{a_{1}}, k_{1}=\frac{h_{1}}{a_{1}}, u_{0}=N_{20}+\frac{h_{2}}{a_{2}}, u_{1}=\frac{h_{2}}{a_{2}} \\
A=\left[\frac{a_{12} k_{1} u_{1}+a_{11} k_{0}^{2}-a_{11} k_{1}^{2}}{a_{1}}\right]+\left[\frac{a_{12} k_{1} u_{0}}{a_{2}-a_{1}}\right]-\left[\frac{a_{12} k_{0} u_{0}}{a_{2}}\right]
\end{gathered}
$$

\section{Results and Discussions}

The variation of $N_{1}$ and $N_{2}$ verses time t in the interval [0, 1] is computed analytically employing Homotopy perturbation method for a wide range of values of the characterizing the parameters $a_{1}, a_{2}, a_{11}, a_{12}$ and $a_{22}$ as shown in Table-1. From Fig.1-8, it is observed that when the growth rate of host increases the $N_{2}$ curves are falling with increasing steepness. Both $N_{1}$ and $N_{2}$ are decreases with time $t$ because of utilization of energy. For $a_{12}<1$, weak commensalism of host over commensal $N_{2}$ falls with slower rate than $N_{1}$. However with increasing growth rate of host steepness increase for the $N_{2}$ curves and falling rate is decreased comparison with $N_{1}$. With increasing $a_{22}$ steepness decreases.

For $a_{12}=1$, the steepness of $N_{2}$ increases with $a_{2}$ however falling of $N_{2}$ is faster along with increasing $a_{22}$ than $N_{1}$. For $a_{12}>1$, strong commensalism of host over commensal it is observed that $N_{1}$ increasing to some extent and then start falling. This tendency is observed at slow rate in case of $a_{12}=1$. Because of growth rate of the specie $S_{1}, N_{1}$ falls much faster than $N_{2}$. However this fall becomes slower in case of $a_{12}>1$.

\section{Conclusion}

Investigate some relation-chains between the species such as commensal-host ecological model with replenishment rate between two species $\left(S_{1}, S_{2}\right)$ with the population relations. The present paper deals with an investigation on analytical approach of a typical two species syn eco-system. The system of coupled non-linear differential equations of populations of two species $S_{1}$ and $S_{2}$ has been solved analytically using Homotopy perturbation method. Our analytical results are compared with previous work and the results are good agreement with the previous work. This method can be extended to solve a mathematical model of a population of three species and four species with SynEcological system.

\section{Nomenclature}

$\begin{array}{ll}\text { Symbol } & \text { Meaning } \\ N_{1} & \text { Population of the specie } S_{1} \\ N_{2} & \text { Population of the specie } S_{2} \\ a_{1} & \text { Natural growth rate of the specie } S_{1} \\ a_{2} & \text { Natural growth rate of the specie } S_{2} \\ a_{11} & \text { Rate of decrease of the specie } S_{1} \\ a_{22} & \text { Rate of decrease of the specie } S_{2} \\ a_{12} & \text { Inhibition coefficient } \\ h_{1} & \text { Replenishment rate of the specie } S_{1} \\ h_{2} & \text { Replenishment rate of the specie } S_{1}\end{array}$

\section{Appendix A}

\section{Basic Concept of the Homotopy Perturbation Method (HPM) [9-16]}

To explain this method, let us consider the following function:

$$
\mathrm{D}_{\mathrm{o}}(\mathrm{u})-\mathrm{f}(\mathrm{r})=0, \quad \mathrm{r} \in \Omega
$$

with the boundary conditions of

$$
\mathrm{B}_{\mathrm{o}}\left(\mathrm{u}, \frac{\partial \mathrm{u}}{\partial \mathrm{n}}\right)=0, \quad \mathrm{r} \in \Gamma
$$

where $D_{o}$ is a general differential operator, $B_{o}$ is a boundary operator, $f(r)$ is a known analytical function and $\Gamma$ is the boundary of the domain $\Omega$. In general, the operator $D_{o}$ can be divided into a linear part $L$ and a non-linear $\operatorname{part}_{N}$. The eqn. (A.1) can therefore be written as 


$$
L(u)+N(u)-f(r)=0
$$

By the Homotopy technique, we construct a Homotopy $v(r, p): \Omega \times[0,1] \rightarrow \Re$ that satisfies

$$
\begin{gathered}
H(v, p)=(1-p)\left[L(v)-L\left(u_{0}\right)\right]+p\left[D_{o}(v)-f(r)\right]=0 . \\
H(v, p)=L(v)-L\left(u_{0}\right)+p L\left(u_{0}\right)+p[N(v)-f(r)]=0 .
\end{gathered}
$$

where $p \in[0,1]$ is an embedding parameter, and $u_{0}$ is an initial approximation of eqn. (A.1) that satisfies the boundary conditions. From eqns. (A.4) and (A.5), we have

$$
\begin{aligned}
& H(v, 0)=L(v)-L\left(u_{0}\right)=0 \\
& H(v, 1)=D_{o}(v)-f(r)=0
\end{aligned}
$$

When $p=0$, the eqns. (A.4) and (A.5) become linear equations. When $p=1$, they become non-linear equations. The process of changing $p$ from zero to unity is that of $L(v)-L\left(u_{0}\right)=0$ to $D_{o}(v)-f(r)=0$. We first use the embedding parameter $p$ as a "small parameter" and assume that the solutions of eqns. (A.4) and (A.5) can be written as a power series in $p$ :

$$
v=v_{0}+p v_{1}+p^{2} v_{2}+\ldots
$$

Setting $p=1$ results in the approximate solution of the eqn. (A.1):

$$
\begin{gathered}
(1-p)\left[\frac{d\left(N_{1_{0}}+p N_{1_{1}}+p^{2} N_{1_{2}}+\ldots .\right)}{d t}-a_{1}\left(N_{1_{0}}+p N_{1_{1}}+p^{2} N_{1_{2}}+\ldots .\right)-h_{1}\right]+ \\
p\left[\begin{array}{l}
\left.\frac{d\left(N_{1_{0}}+p N_{11}+p^{2} N_{1_{2}}+\ldots .\right)}{d t}-a_{1}\left(N_{1_{0}}+p N_{1_{1}}+p^{2} N_{1_{2}}+\ldots .\right)-h_{1}\right] \\
+a_{11}\left(N_{1_{0}}+p N_{11}+p^{2} N_{1_{2}}+\ldots .\right) \\
-a_{12}\left(N_{1_{0}}+p N_{11}+p^{2} N_{1_{2}}+\ldots\right)\left(N_{2_{0}}+p N_{2_{1}}+p^{2} N_{2_{2}}+\ldots .\right)
\end{array}\right]=0 \\
(1-p)\left[\begin{array}{l}
\frac{d\left(N_{2_{0}}+p N_{2_{1}}+p^{2} N_{2_{2}}+\ldots .\right)}{d t}-a_{2}\left(N_{2_{0}}+p N_{2_{1}}+p^{2} N_{1_{2}}+\ldots .\right)-h_{2} \\
d t
\end{array}\right]+ \\
p\left[\begin{array}{l}
\frac{d\left(N_{2_{0}}+p N_{2_{1}}+p^{2} N_{2_{2}}+\ldots .\right)}{d t}-a_{2}\left(N_{2_{0}}+p N_{2_{1}}+p^{2} N_{2_{2}}+\ldots .\right)-h_{2} \\
+a_{22}\left(N_{2_{0}}+p N_{2_{1}}+p^{2} N_{2_{2}}+\ldots .\right)^{2}
\end{array}\right]=0
\end{gathered}
$$

Comparing the coefficients of like powers of $p$ in (B.5) and (B.6) we get

$$
\begin{gathered}
p^{0}: \frac{d N_{1_{0}}}{d t}-a_{1} N_{1_{0}}-h_{1}=0 \\
p^{0}: \frac{d N_{2} 0}{d t}-a_{2} N_{2_{0}}-h_{2}=0 \\
p^{1}: \frac{d N_{1_{1}}}{d t}-a_{1} N_{1_{1}}+a_{11} N_{1}^{2}-a_{12} N_{1} N_{2}=0 \\
p^{1}: \frac{d N_{2}}{d t}-a_{2} N_{2_{1}}+a_{22} N_{2}^{2}=0
\end{gathered}
$$

The initial approximations is as follows

$$
\begin{aligned}
& N_{1}(0)=N_{10}, N_{2}(0)=N_{20} \\
& N_{1 i}(0)=N_{2 i}(0)=0, \quad i=1,2,3 \ldots \ldots .
\end{aligned}
$$

Solving the eqns. (B.7) - (B.10) and using the boundary conditions (B.11) and (B.12) we obtain the following results:

$$
N_{1_{0}}(t)=\left(k_{0} e^{a_{1} t}-k_{1}\right)
$$




$$
\begin{gathered}
N_{1_{1}}(t)=A e^{a_{1} t}+\left[\frac{a_{11} k_{1}^{2}-a_{12} k_{1} u_{1}}{a_{1}}\right]+\left[2 a_{11} k_{0} k_{1}-a_{12} k_{0} u_{1}\right] t e^{a_{1} t}-\left[\frac{a_{12} k_{1} u_{0}}{a_{2}-a_{1}}\right] \\
-\left[\frac{a_{11} k_{0}^{2} e^{a_{2} t}}{a_{1}}\right]+\left[\frac{a_{12} k_{0} u_{0} e^{\left(a_{1}+a_{2}\right) t}}{a_{2}}\right] \\
N_{2_{0}}(t)=\left(u_{0} e^{a_{2} t}-u_{1}\right) \\
N_{2_{1}}(t)=\left(\frac{a_{22}}{a_{2}}\right)\left[\left(u_{0}^{2}-u_{1}^{2}\right) e^{a_{2} t}-\left(u_{1}^{2}-u_{0}^{2} e^{2 a_{2} t}\right)\right]+2 a_{22} u_{0} u_{1} e^{a_{2} t}
\end{gathered}
$$

Where $k_{0}, k_{1}, u_{0}, u_{1}$ and $A$ are defined in the text (6) and (7).

According to the HPM, we can conclude that

$$
\begin{aligned}
& N_{1}=\lim _{p \rightarrow 1} N_{1}(t)=N_{1_{0}}+N_{1_{1}} \\
& N_{2}=\lim _{p \rightarrow 1} N_{2}(t)=N_{2_{0}}+N_{2_{1}}
\end{aligned}
$$

After putting the eqns. (B.13) and (B.14) into an eqn. (B.17) and (B.15) and (B.16) into an eqn. (B.18), we obtain the solutions in the text (4) - (7).

\section{Appendix C}

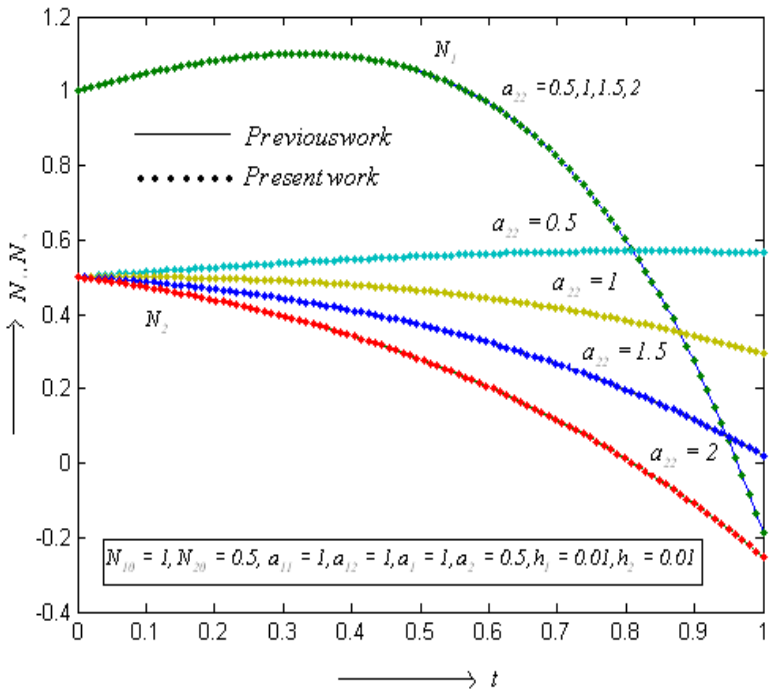

Fig 1. The populations $N_{1}(t)$ and $N_{2}(t)$ versus the time $t$. The populations were computed using the eqns. (4) and (5) for various values of the dimensionless parameters.

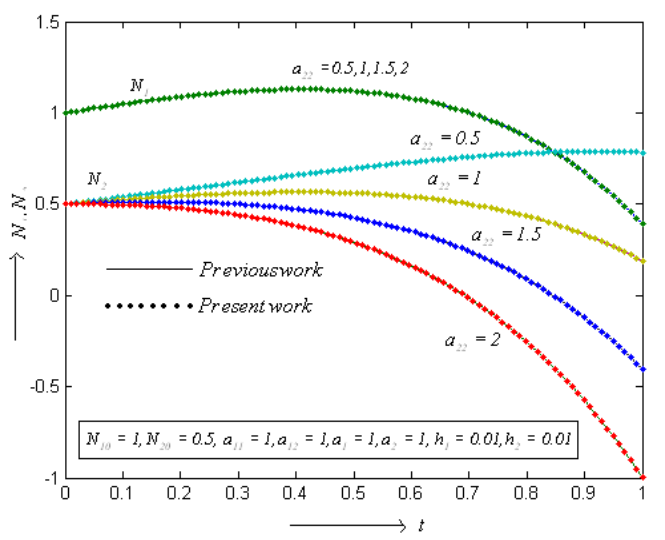

Fig 2. The populations $N_{1}(t)$ and $N_{2}(t)$ versus the time $t$. The populations were computed using the eqns. (4) and (5) for various values of the dimensionless parameters.

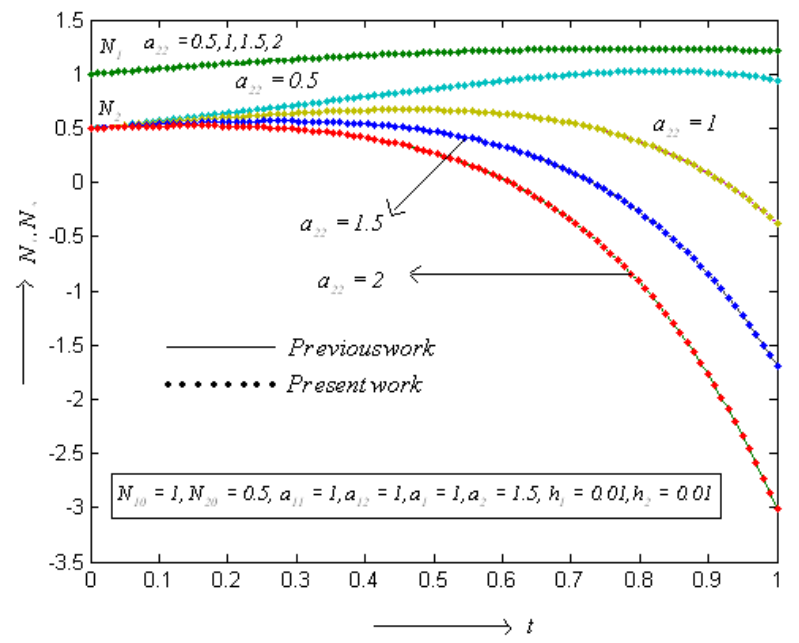

Fig 3. The populations $N_{1}(t)$ and $N_{2}(t)$ versus the time $t$. The populations were computed using the eqns. (4) and (5) for various values of the dimensionless parameters. 


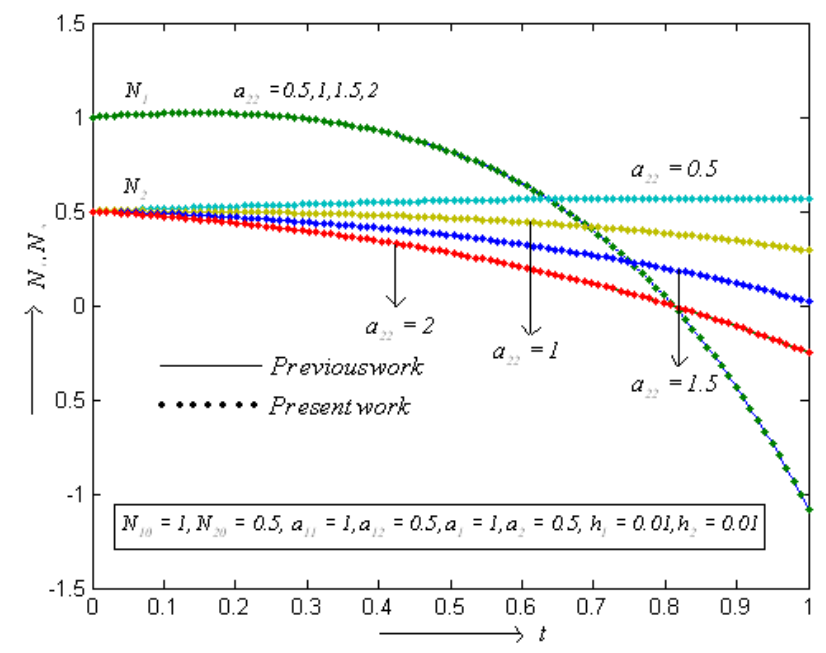

Fig 4. The populations $N_{1}(t)$ and $N_{2}(t)$ versus the time $t$. The populations were computed using the eqns. (4) and (5) for various values of the dimensionless parameters.

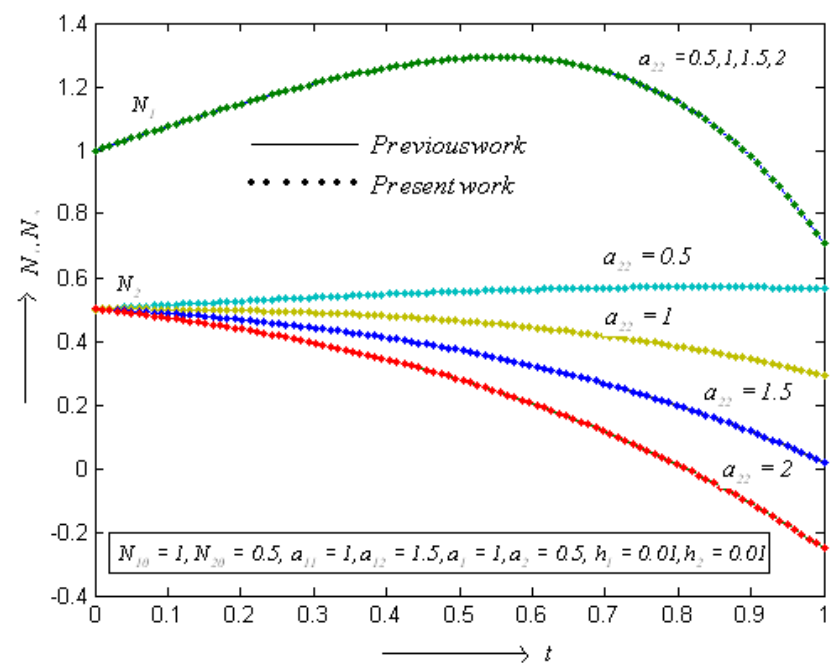

Fig 5. The populations $N_{1}(t)$ and $N_{2}(t)$ versus the time $t$. The populations were computed using the eqns. (4) and (5) for various values of the dimensionless parameters.

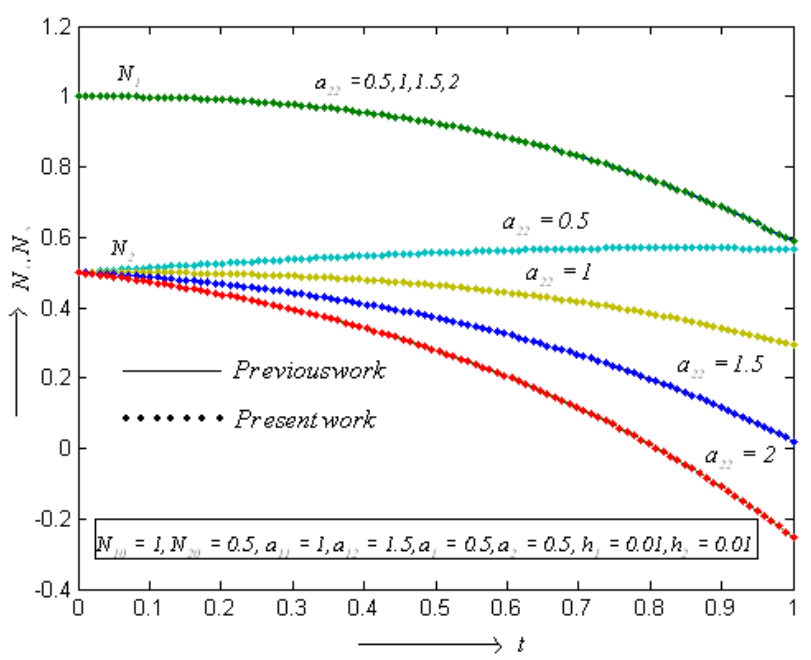

Fig 6. The populations $N_{1}(t)$ and $N_{2}(t)$ versus the time $t$. The populations were computed using the eqns. (4) and (5) for various values of the dimensionless parameters.

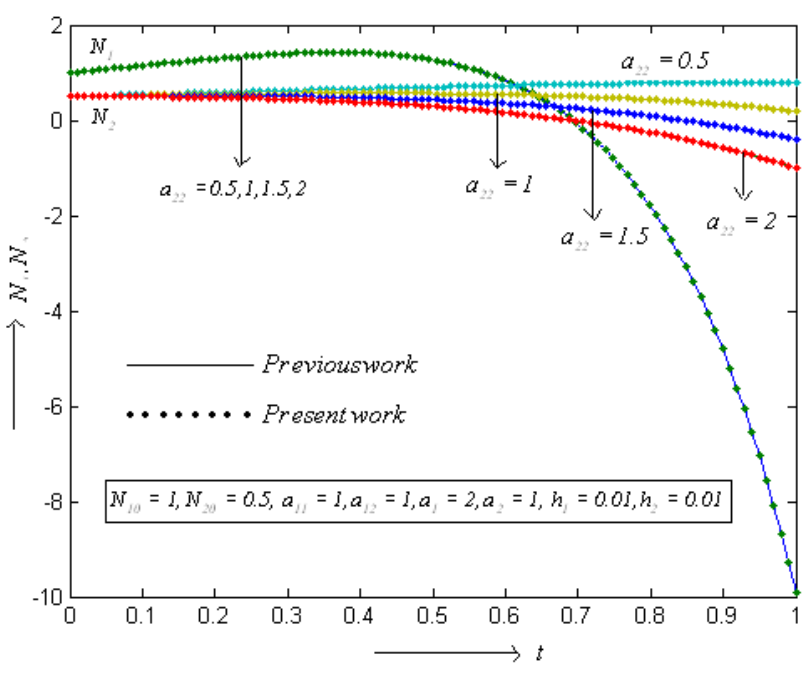

Fig 7. The populations $N_{1}(t)$ and $N_{2}(t)$ versus the time $t$. The populations were computed using the eqns. (4) and (5) for various values of the dimensionless parameters.

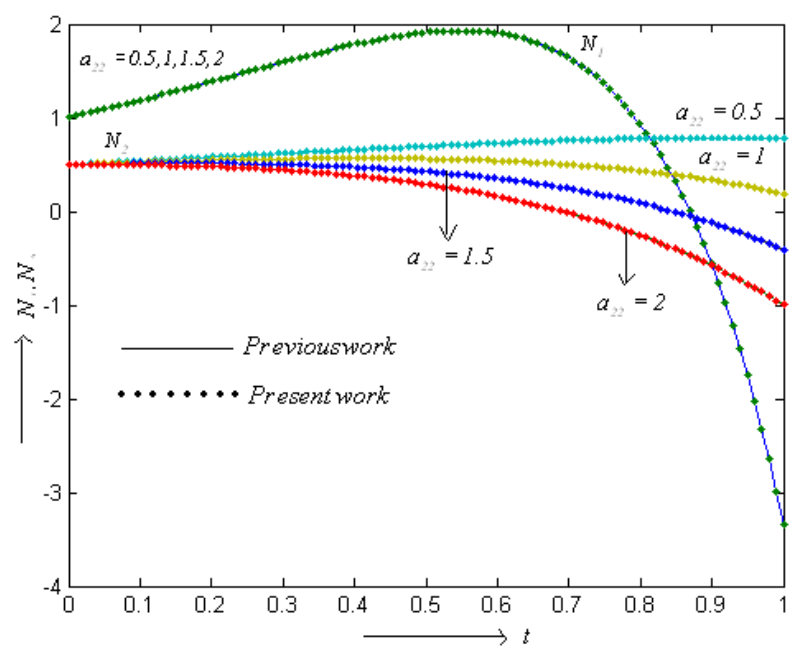

Fig 8. The populations $N_{1}(t)$ and $N_{2}(t)$ versus the time $t$. The populations were computed using the eqns. (4) and (5) for various values of the dimensionless parameters. 
Table 1. Numerical values of the dimensionless parameters for previous and present work.

\begin{tabular}{llllllllll}
\hline 1 & $N_{10}=1$ & $N_{20}=0.5$ & $a_{11}=1$ & $a_{12}=1$ & $a_{1}=1$ & $a_{2}=0.5$ & $h_{1}=0.01$ & $h_{2}=0.01$ & $a_{22}: 0.5,1,1.5,2$ \\
2 & $N_{10}=1$ & $N_{20}=0.5$ & $a_{11}=1$ & $a_{12}=1$ & $a_{1}=1$ & $a_{2}=0.5$ & $h_{1}=0.01$ & $h_{2}=0.01$ & $a_{22}: 0.5,1,1.5,2$ \\
3 & $N_{10}=1$ & $N_{20}=0.5$ & $a_{11}=1$ & $a_{12}=1$ & $a_{1}=1$ & $a_{2}=0.5$ & $h_{1}=0.01$ & $h_{2}=0.01$ & $a_{22}: 0.5,1,1.5,2$ \\
4 & $N_{10}=1$ & $N_{20}=0.5$ & $a_{11}=1$ & $a_{12}=1$ & $a_{1}=1$ & $a_{2}=0.5$ & $h_{1}=0.01$ & $h_{2}=0.01$ & $a_{22}: 0.5,1,1.5,2$ \\
5 & $N_{10}=1$ & $N_{20}=0.5$ & $a_{11}=1$ & $a_{12}=1.5$ & $a_{1}=1$ & $a_{2}=0.5$ & $h_{1}=0.01$ & $h_{2}=0.01$ & $a_{22}: 0.5,1,1.5,2$ \\
6 & $N_{10}=1$ & $N_{20}=0.5$ & $a_{11}=1$ & $a_{12}=1$ & $a_{1}=1$ & $a_{2}=0.5$ & $h_{1}=0.01$ & $h_{2}=0.01$ & $a_{22}: 0.5,1,1.5,2$ \\
7 & $N_{10}=1$ & $N_{20}=0.5$ & $a_{11}=1$ & $a_{12}=1$ & $a_{1}=1$ & $a_{2}=0.5$ & $h_{1}=0.01$ & $h_{2}=0.01$ & $a_{22}: 0.5,1,1.5,2$ \\
8 & $N_{10}=1$ & $N_{20}=0.5$ & $a_{11}=1$ & $a_{12}=1$ & $a_{1}=1$ & $a_{2}=0.5$ & $h_{1}=0.01$ & $h_{2}=0.01$ & $a_{22}: 0.5,1,1.5,2$ \\
\hline
\end{tabular}

\section{Acknowledgement}

The authors are thankful to Shri. S. Natanagopal, Secretary, The Madura College Board, Dr. R. Murali, The Principal and Mr. S. Muthukumar, Head of the Department of Mathematics, The Madura College (Autonomous), Madurai, Tamil Nadu, India for their constant encouragement.

\section{References}

[1] N. Phani Kumar, C. Srinivasa kumar and N.Ch. Pattabhi Ramacharyulu, "A recursive procedure for commensal-host ecological model with replenishment rate for both the speciesA numerical approach, ARPN Journal of Engineering and Applied Sciences, Vol. 5, No.10 (2010).

[2] B. Bhaskara Rama Sarma. N.Ch Pattabhi Ramacharyulu and S.V.N. Lalitha, "A recursive procedure for two species competing eco system with decay and replenishment for one specie”, Acta ciencia Indica. Xxxv M. (2): 487-496 (2009).

[3] J.M. Cushing, "Integro - differential equations and delay models in population dynamics", Lect. Notes in Biomathematics. Vol. 20, springer - reflag, Heidelbegh 9.(1977).

[4] J.N. Kapur, "Mathematical models in biology and medicine", Affiliated East-West 9, (1985).

[5] W.E. Langlois and R.S. Rivlin, "Steady flow of slightly viscoelastic fluids", (Doctoral thesis of W-Elanglois), Brown universality, (1957).

[6] W.J. Meyer, "Concepts of mathematical modeling", McGrawhill, (1985).

[7] N. Phanikumar and N. Ch. Pattabhi Ramacharyulu, "On the stability of a commesal-host harvested species pair with limited resources", Communicated to International journal of computational cognition.

[8] N.C. Srinvias, "Some mathematical aspects of modeling in bio-medical sciences", Ph.D. thesis, submitted to Kakatiya university, (1991).
[9] Q.K. Ghori, M. Ahmed, and A. M. Siddiqui, "Application of Homotopy perturbation method to squeezing flow of a newtonian fluid", Int. J. Non-linear Sci. Numer. Simulat, 8 179-184 (2007).

[10] T. Ozis, and A. Yildirim, "A comparative study of He's Homotopy perturbation method for determining frequencyamplitude relation of a non-linear oscillator with discontinuities", Int. J. Nonlinear Sci. Numer. Simulat, 8, 243248 (2007).

[11] S. J. Li, and Y. X. Liu, "An improved approach to non-linear dynamical system identification using PID neural networks", Int. J. Non-linear Sci. Numer. Simulat, 7, 177-182 (2006).

[12] M. M. Mousa, S. F. Ragab, and Z. Nturforsch, "Application of the Homotopy perturbation method to linear and non-linear schrödinger equations",. Zeitschrift für naturforschung", 63, 140-144 (2008).

[13] J.H. He, "Homotopy perturbation technique", Comp Meth. Appl. Mech. Eng, 178, 257-262 (1999).

[14] J. H. He, "Homotopy perturbation method: a new non-linear analytical technique", Appl. Math. Comput, 135, 73-79(2003).

[15] J. H. He, "A simple perturbation approach to blasius equation", Appl. Math. Comput, 140, 217-222 (2003).

[16] P.D. Ariel "Alternative approaches to construction of Homotopy perturbation algorithms", Nonlinear. Sci. Letts. A., 1, 43-52 (2010).

[17] V. Ananthaswamy, and L. Rajendran, "Analytical solution of non-linear kinetic equation in a porous pellet", Global journal of pure and applied mathematics, Vol.8, no. 2, 101-111 (2012).

[18] V. Ananthaswamy and L. Rajendran, "Analytical solution of two-point non-linear boundary value problems in a porous catalyst particles", International Journal of Mathematical Archive, 3 (3), 810-821 (2012).

[19] V.Ananthaswamy and L. Rajendran, "Analytical solution of non-isothermal diffusion-reaction processes and effectiveness factors", ISRN Physical Chemistry, Hindawi publishing corporation, 2013, Article ID 487240, 1-14 (2013). 